\title{
Verfahren und Programm
}

\author{
Angelika Martin
}

\begin{abstract}
Das Unabhängige Landeszentrum für Datenschutz hat aus einer Anfrage eines Behördenmitarbeiters im Umgang mit dem Verfahrensverzeichnis folgende FAQ entwickelt.
\end{abstract}

\section{Frage:}

Was unterscheidet eigentlich ein Verfahren von einem Programm (oder Software oder Fachverfahren)? Ist ein automatisiertes Verfahren das gleiche wie ein Programm oder was macht ein Verfahren zusätzlich aus?

\section{Antwort:}

Zunächst einmal die Definition eines automatisierten Verfahrens:

Ein automatisiertes Verfahren im Sinne des LDSG ist eine Verwendung von (personenbezogenen) Daten

zu einem bestimmten Zweck

- mit Unterstützung von informationstechnischen Geräten (Hardware)

und Computerprogrammen (Software)

- eingebunden in ein organisatorisches Regelwerk.

Sie haben recht: Betrachtet man manche große Fachverfahren, wie z.B. ein Fachverfahren zur Verwaltung von Einwohnermeldedaten, dann unterscheidet sich das Verfahren (Verfahren: Verarbeitung von Einwohnermeldedaten) nicht von dem eingesetzten Fachverfahren $($ Programm $=$ Software).

Wendet man die oben genannte Definition auf dieses Beispiel an, dann gilt Folgendes:

\section{Fall A}

Zweck: Verwaltung und Verarbeitung von Einwohnermeldedaten

- Hardware: hauptsächlich der Server, Zugriff der Clients über das Netz

- Software: es wird nur ein Fachverfahren (Programm xy) eingesetzt

- Regelwerk: nur autorisierte Personen dürfen die Daten einsehen/verarbeiten.

In diesem Fall werden Sie Ihrer Verfahrensakte nur ein Fachverfahren/Programm dokumentieren.
Doch es ist durchaus möglich, dass zur Erfüllung eines bestimmten Verfahrenszwecks auch mehrere Fachverfahren/Programme notwendig sind. Gehen wir von folgendem Beispiel aus (schon angelehnt an die oben genannte Definition):

\section{Fall B}

Z Zweck: Kommunale Finanzverwaltung

- Hardware: hauptsächlich der Server, Zugriff der Clients über das Netz

- Software: Einsatz von zwei Fachverfahren/Programmen (Programm ab $+c d$ )

- Regelwerk: bei beiden Fachverfahren eine Benutzerverwaltung

In diesem Beispiel wird z.B. das Fachverfahren ,ab" zur Verwaltung der Finanzdaten eingesetzt und das Fachverfahren ,cd“ nur zum Abruf der Daten. Beide Programme werden aber eingesetzt, um den Verfahrenszweck „Kommunale Finanzverwaltung“ zu erfüllen. In diesem Fall werden Sie in Ihrer Verfahrensakte zwei Fachverfahren/Programme dokumentieren.

Betrachten wir noch ein drittes Beispiel, bei dem zur Erfüllung des Verfahrenszwecks mehrere Programme/Fachverfahren eingesetzt werden:

\section{Fall C}

Zweck: Allgemeine Bürokommunikation

- Hardware: hauptsächlich der Server, Zugriff der Clients über das Netz

- Software: Standardsoftware Word, Excel und Outlook, ein selbstentwickeltes Registraturprogramm und ein Programm zur Archivierung

- Regelwerk: auf das Registratur- und Archivierungsprogramm dürfen nur autorisierte Personen zugreifen, auf die Standardsoftware alle Personen

In diesem Fall werden Sie in Ihrer Verfahrensakte fünf unterschiedliche Programme/Fachverfahren dokumentieren.

Um die Verfahren in Ihrer Behörde zu identifizieren, sollten Sie genau nach dem beschriebenen Schema vorgehen - am besten in Zusammenarbeit mit dem/der Datenschutzbeauftragten sowie Vertretern der Administration und der Fachbereiche:
1. Identifizierung des Zwecks der Datenverarbeitung (z.B. angelehnt an die Aufgabenbereiche, die die Behörde wahrnimmt).

2. Berücksichtigung der benötigten Hardware.

3. Überlegung, welche Fachverfahren/Programme eingesetzt werden müssen.

4. Überlegung, wer wann wie und in welchem Umfang auf die Daten zugreifen darf.

\section{Frage:}

Muss eine Daten verarbeitende Stelle in Schlewig-Holstein zusätzlich zu dem Verfahrensverzeichnis auch ein Programmverzeichnis führen?

\section{Antwort:}

Ja. Nach $\S 8$ Datenschutzverordnung muss jede Daten verarbeitende Stelle neben dem Verfahrensverzeichnis (§ 7 LDSG SH) auch ein Programm- und Geräteverzeichnis bzw. ein entsprechendes Inventarverzeichnis führen. Eine vergleichbare Verpflichtung ergibt sich aus dem Landeshaushaltsrecht.

\section{Frage}

Welche Informationen kommen in das Verfahrensverzeichnis und welche in das Programmverzeichnis?

\section{Antwort}

Im Verfahrensverzeichnis werden die im § 7 LDSG SH aufgeführten Angaben dokumentiert. Im Programmverzeichnis muss die Bezeichnung des Programms und die Bezeichnung des Gerätes, auf dem das Programm gespeichert ist, dokumentiert werden. Programm- und Geräteverzeichnis bzw. Inventarverzeichnis sind nur eine Bestandsliste.
Angelika Martin
Unabhängiges Landeszentrum für
Datenschutz Schleswig-Holstein
Holstenstraße 98, $24103 \mathrm{Kiel}$
Tel ++49-431-988-1280, Fax-1223,
E-Mail:LD34@datenschutzzentrum.de
http://www.datenschutzzentrum.de 